\title{
Estrategias de internacionalización en los grupos de investigación: un estudio de caso
}

\author{
María Paz López
}

* Profesora en Ciencias de la Educación, Becaria del Consejo Nacional de Investigaciones Científicas y Técnicas, Investigadora del Centro de Estudios Interdisciplinarios en Problemáticas Internacionales y Locales de la Universidad Nacional del Centro de la Provincia de Buenos Aires, Buenos Aires, Argentina. Correo electrónico: mpaz_lo@yahoo.com.ar

Recibido: 28 de agosto de 2013 Aprobado: 3 de mayo de 2014

Cómo citar este artículo: López, M. P. (2014). Estrategias de internacionalización en los grupos de investigación: un estudio de caso. Memorias, 12(21), 133-143. doi: http:// dx.doi.org/10.16925/me.v12i21.864

\begin{abstract}
Resumen
Introducción: la internacionalización científica en los grupos de investigación es abordada aquí en cuanto a estrategias de internacionalización. Primero, se proponen y definen los conceptos de grupo de investigación, estrategias de internacionalización, objetivos de producción de conocimientos y orientación de la investigación. Segundo, se describen y analizan los resultados de investigación obtenidos entre el 2011 y el 2012 a partir del análisis de un grupo de investigación en Ciencias Veterinarias de una universidad argentina. Metodología: se realizó un estudio de corte cualitativo, basado en la realización de entrevistas a los responsables del grupo de investigación y en el análisis de documentos institucionales de este. Resultados: se indicó la existencia de dos estrategias principales de internacionalización en el grupo de investigación estudiado, por un lado, las de articulación entre los objetivos de la producción de conocimiento y la orientación de la investigación, y por otro, las de complementación entre los mecanismos de financiamiento institucionalizados y los recursos adicionales generados por el grupo. Conclusiones: se evidencia una conciencia sobre la importancia de la internacionalización en el grupo de investigación dentro del marco de producción de conocimiento y consecución de recursos; no obstante, existen diferencias con respecto al momento de la orientación de producción de conocimiento enfocada al ámbito internacional o local.
\end{abstract}

Palabras clave: estrategias de internacionalización, grupo de investigación, producción de conocimiento, política científica.

\section{Internationalization Strategies in Research Groups: A Case Study}

\begin{abstract}
Introduction: Scientific internationalization of research groups is addressed here in terms of internationalization strategies. First, the concept of research group, internationalization strategies, knowledge production goals and research orientation are proposed and defined. Secondly, the results of research obtained between 2011 and el 2012 are described and analyzed based on the analysis of a Veterinary Sciences research group at an Argentine university. Methodology: A qualitative type study was carried out, based on interviews with those responsible for a research group and analysis of that same group's institutional documents. Results: Two main internationalization strategies were found in the research group studied; on the one hand, those involving coordination between the goals for knowledge production and orientation of the research, and on the other hand, complementation between institutionalized financing mechanisms and additional funds generated by the group. Conclusions: There is evidence of awareness of the importance of internationalization in the research group in the framework of knowledge production and fundraising; however, there are differences regarding the time for orientation of knowledge production focused on the international or local sphere.
\end{abstract}

Keywords: internationalization strategies, research group, knowledge production, scientific policy.

\section{Estratégias de internacionalização nos grupos de pesquisa: um estudo de caso}

\section{Resumo}

Introdução: a internacionalização científica nos grupos de pesquisa é abordada aqui em termos de estratégias de internacionalização. Primeiramente, são propostos e definidos os conceitos de grupo de pesquisa, estratégias de internacionalização, objetivos de produção de conhecimentos e orientação da pesquisa. Em seguida, são descritos e analisados os resultados de pesquisa obtidos entre 2011 e 2012 a partir da análise de um grupo de pesquisa em Ciências Veterinárias de uma universidade argentina. Metodologia: realizou-se um estudo de corte qualitativo, baseado na realização de entrevistas aos responsáveis pelo grupo de pesquisa e na análise de documentos institucionais deste. Resultados: indicou-se a existência de duas estratégias principais de internacionalização no grupo de pesquisa estudado; por um lado, as de articulação entre os objetivos da produção de conhecimento e a orientação da pesquisa; por outro, as de complementação entre os mecanismos de financiamento institucionalizados e os recursos adicionais gerados pelo grupo. Conclusões: evidencia-se uma consciência sobre a importância da internacionalização no grupo de pesquisa dentro do marco de produção de conhecimento e consecução de recursos; contudo, existem diferenças a respeito do momento da orientação de produção de conhecimento enfocada no âmbito internacional ou local.

Palavras-chave: estratégias de internacionalização, grupo de pesquisa, produção de conhecimento, política científica. 


\section{Introducción ${ }^{1}$}

La propia naturaleza de los procesos de investigación y difusión de los conocimientos científicos, así como la dinámica de formación de investigadores, hacen de la internacionalización una práctica intrínseca a la actividad científica (Red Iberoamericana de Indicadores de Ciencia y Tecnología [RICYT], 2007). La revisión de la literatura reciente, en el marco de los Estudios Sociales de la Ciencia y la Tecnología, indica que la internacionalización científica de América Latina en general, y Argentina en particular, es cada vez más frecuentemente objeto de mediciones (Sebastián, 2003), a la vez que las modalidades y consecuencias de esta para la producción de conocimiento en la región se analizan desde una perspectiva crecientemente crítica, es decir, teniendo en cuenta tanto los aspectos positivos como negativos de esta (Kreimer y Levin, 2011).

Distintos aportes referidos a la internacionalización de los grupos de investigación de América Latina en general, y Argentina en particular, indican una variedad de consecuencias para la producción de conocimientos y el desarrollo económico y social de los países. En este sentido, la internacionalización permite el acceso a importantes recursos económicos, la inserción de los científicos en las discusiones vigentes en el ámbito internacional, la apertura de nuevas líneas de investigación y la formación de recursos humanos, el aumento del prestigio local, el acceso a nuevos espacios de publicación, aunque también puede generar la estipulación exógena de las agendas de investigación y de las metodologías de trabajo, la fuga de cerebros, la desconexión de los conocimientos producidos en las redes internacionales respecto de las problemáticas prioritarias nacionales (Gaillard, 1994; Licha, 1996; Vessuri, 1998; Kreimer, 2006).

En este marco, el presente artículo busca contribuir al estudio de la internacionalización de la producción de conocimientos proponiendo un análisis centrado en las estrategias de los grupos de investigación. En tal sentido, las preguntas que guían el trabajo son: ¿cuáles son las estrategias de internacionalización que los grupos de investigación ponen en marcha en relación con los objetivos de la producción de conocimientos? ¿Qué estrategias de internacionalización

Artículo de investigación resultado del trabajo sobre las estrategias de internacionalización de un grupo de investigación en Ciencias Veterinarias de una universidad argentina. desarrollan los grupos de investigación en relación con la evaluación de los organismos de ciencia y tecnología y las fuentes de financiamiento disponibles en el ámbito nacional? ¿Qué estrategias llevan adelante los grupos de investigación para internacionalizarse en el marco de la pluralidad de dimensiones implicadas en la actividad de investigación? El trabajo describe y analiza los resultados obtenidos en una investigación llevada adelante entre los años 2011 y 2012 en un grupo de investigación en Ciencias Veterinarias de una universidad de Argentina.

Se considera importante desarrollar análisis en el nivel de los grupos de investigación, entendiendo que estos, a través de sus estrategias, son los que cotidianamente desarrollan la tarea de producción de conocimientos, a la vez que influyen en la interpretación y el delineamiento de las políticas públicas en la materia (Dagnino, 2007). ${ }^{2}$ A su vez, las diversas orientaciones adoptadas por las actividades científicas y tecnológicas tienen implicancias respecto al uso social del conocimiento y, más ampliamente, en el desarrollo económico, político y social (Albornoz, 2009).

\section{Grupos de investigación y estrategias de internacionalización}

El presente trabajo reconoce la naturaleza colectiva de la producción de conocimiento desarrollada en ámbitos académicos; más precisamente, entiende que el grupo de investigación constituye la mínima unidad espacio-temporal de producción de conocimiento de carácter colectivo (Bianco y Sutz, 2005). En este marco, considera los grupos de investigación como ámbitos de identificación cultural, política, cognoscitiva e institucional, que se van estructurando históricamente a través de relaciones intergeneracionales, en los lugares de trabajo y en los diferentes ámbitos de actuación institucional (Kreimer, 2000).

A partir del proceso de interacción, los grupos de investigación desarrollan diversas vertientes identitarias referidas a las temáticas trabajadas, la trayectoria de trabajo común y el sentido de pertenencia al colectivo (Bianco y Sutz, 2005). A su vez, hay que tener en cuenta la múltiple pertenencia institucional de los

\footnotetext{
2 Por supuesto, se contempla la existencia de condiciones estructurales que influyen a -y son influidas por- los grupos de investigación, entre las que se destacan la pertenencia disciplinar y las instituciones en las cuales se insertan los mismos, ya sean las universidades u otras agencias de ciencia y tecnología (Clark, 1991).
} 
grupos de investigación, los cuales pueden estar no sólo considerados por las políticas propias de la universidad, sino también por otros organismos estatales que cuentan con becas de formación y carrera científica, así como con subsidios para la investigación.

Las trayectorias recorridas por los grupos de investigación incluyen, aunque no como un recurso exclusivo, la actuación de científicos provenientes de otros países, a través de la participación en proyectos conjuntos de investigación, la movilidad científica internacional, las publicaciones con autoría internacional, entre otros (Kreimer, 2000; De Filippo, Morillo y Fernández, 2008; Vessuri, 2009). Asimismo, este trabajo considera la importancia de incorporar la publicación en el extranjero como expresión de la internacionalización de los grupos de investigación (Plaza y Bordons, 2006). Por otra parte, la internacionalización puede ser entendida como parte de las exigencias institucionales para la formación de recursos humanos, el reconocimiento y difusión de las actividades realizadas y la calidad de la investigación (RICYT, 2007).

Tal como se mencionó anteriormente, el presente artículo explora las estrategias de internacionalización puestas en marcha por los grupos de investigación. Cabe destacar que la conceptualización del término estrategia pretende apartarse del pensamiento sociológico que tiende a asociarla únicamente a la conducta racional, consciente y deliberada de los sujetos. Más precisamente, entiende las estrategias en un sentido próximo al propuesto por Bourdieu, como acciones y decisiones basadas en el sentido práctico de los agentes, con diferentes grados de deliberación y razonamiento (Bourdieu, 1987; Wilkis, 2004). Así, las estrategias de internacionalización son entendidas como "el conjunto de decisiones, acciones, inversiones e interpretaciones que realizan los grupos de investigación sobre sus posibilidades y oportunidades en el contexto de situación" (Vaccarezza y Zabala, 2002, p. 31), referidas a la internacionalización de sus actividades, a saber: la formación y perfeccionamiento de recursos humanos, la publicación y difusión de resultados, el desarrollo de proyectos de investigación o la obtención de recursos.

El análisis distingue aquellas estrategias relacionadas con los objetivos de la producción de conocimiento considerados como centrales por el grupo, así como aquellas orientadas a responder a las exigencias de las instituciones de ciencia y tecnología en las cuales los grupos de investigación se insertan. Asimismo, el trabajo enfatiza las estrategias desarrolladas con el fin de articular o complementar los objetivos de la producción de conocimiento y la orientación de la investigación en los grupos de investigación.

\section{Estrategias de internacionalización y objetivos de la producción de conocimientos}

Uno de los aspectos centrales que construye la identidad de un grupo de investigación es el tipo de objetivos compartidos respecto de la producción de conocimientos. Esta dimensión diferencia grupos de investigación puramente académicos de aquellos que trabajan en conexión con sectores productivos o de servicios (Bianco y Sutz, 2005). Al respecto, se ha señalado que sólo algunos grupos e investigadores buscan publicar en revistas de reconocimiento internacional, demostrar relaciones fluidas con importantes centros internacionales de la disciplina y obtener premios internacionales, con el fin de constituirse en referentes destacados de la disciplina objeto de estudio (Vaccarezza, 2000).

Este aspecto se vincula con la tramitación simbólica que realizan los grupos de investigación respecto de la tensión que significa la producción de conocimientos desde una patria determinada y un sentimiento paralelo de pertenencia a una comunidad científica internacional, por la cual se gestan mentalidades cosmopolitas y anticosmopolitas entre los científicos (Vessuri, 1991). Mientras que los primeros buscan reconocimiento de los pares en la comunidad científica internacional, los segundos se identifican con el medio local y la resolución de sus problemas. De igual manera, se distinguen grupos orientados al desarrollo de vinculaciones con el medio local y grupos que priorizan las relaciones con actores internacionales (Kreimer y Ugartemendía, 2007).

A su vez, los objetivos de la producción de conocimiento direccionan, potencian o desalientan las estrategias relacionadas con la obtención y utilización de recursos cognitivos, humanos, relacionales y materiales necesarios para insertarse en el ámbito internacional. Además, es preciso tener en cuenta el comportamiento de la comunidad de investigación como actor político en el proceso de formulación e implementación de las políticas científicas. En estos procesos se ponen en juego los intereses específicos, las ventajas relativas, el control de recursos y las visiones ideológicas de la comunidad de investigación sobre la ciencia (Dagnino, 2007). 


\section{Estrategias de internacionalización y orientación de la investigación}

La orientación de la investigación hace referencia a los criterios con los cuales los grupos de investigación entienden que son evaluados en las distintas instituciones a las que se hallan adscritos. Los criterios de evaluación se transforman en señales que "indican a los investigadores a qué dedicar su tiempo para adquirir los atributos por los cuales luego serán juzgados" (Sebastián, 2003, p. 32). En este sentido, la internacionalización puede ser entendida como parte de las exigencias institucionales en las diferentes actividades comprendidas por la producción de conocimientos, a saber: la formación de recursos humanos, el reconocimiento y difusión de las actividades realizadas y la calidad de la investigación (RICYT, 2007).

Otro punto central, desde la orientación de la investigación, es el acceso a fondos para el financiamiento de sus actividades de investigación. Resulta interesante el aporte realizado por Vaccarezza (2000) acerca de la dinámica de captación de recursos para la investigación en el ámbito académico; más precisamente, se destacan los conceptos de rutinas de recursos (o mecanismos de asignación de recursos institucionalizados, como subsidios para proyectos de investigación, programas de becas y otros) y dispositivos de recursos (o recursos adicionales generados por los propios grupos de investigación, como la realización de cursos de especialización para profesionales). A este respecto, puede plantearse una complementación entre los recursos brindados por las rutinas nacionales para responder a las necesidades de la investigación y la construcción de dispositivos de recursos en el ámbito internacional (Luchilo y Guber, 2007; Hubert y Spivak, 2009).

La perspectiva de los grupos de investigación sobre las exigencias institucionales, al igual que los objetivos de la producción de conocimiento, direccionan, potencian o desalientan las estrategias de obtención y uso de recursos cognitivos, humanos, relacionales y materiales necesarios para insertarse en el ámbito internacional. Una vez más es preciso tener en cuenta el comportamiento de la comunidad de investigación como actor político. Es de resaltar que los investigadores, al menos aquellos con influencias en las instancias de formulación e implementación de políticas institucionales, pueden aportar a la definición de las orientaciones de la investigación y la asignación de rutinas de recursos impulsando o desestimando la internacionalización de las actividades de investigación.

\section{Estrategias de internacionalización en un grupo de investigación en Ciencias Veterinarias de una universidad argentina}

\section{Una breve introducción al caso estudiado}

Tal como se ha mencionado anteriormente, el presente artículo describe y analiza los resultados obtenidos de una investigación cualitativa llevada adelante en un grupo de investigación en ciencias veterinarias de una universidad argentina entre los años 2011 y 2012. Es pertinente señalar que la universidad de la cual hace parte el grupo en estudio organiza su sistema científico-tecnológico en grupos de investigación denominados Núcleos de Actividades Científicas y Tecnológicas (NACT), ${ }^{3}$ lo cual facilita el proceso de identificación de las unidades de análisis. Particularmente, el grupo de investigación seleccionado funciona dentro de la Facultad de Ciencias Veterinarias de la universidad, contando con un director y una planta estable de investigadores. A los fines de este artículo, lo denominaremos "Grupo Veterinarias".

El NaCT estudiado constituye uno de los grupos pioneros en la consolidación de la investigación de dicha universidad y presenta importantes indicadores de internacionalización, como la formación de recursos humanos en el extranjero, la participación en programas de cooperación internacional, la colaboración en proyectos de investigación financiados por organizaciones internacionales sin fines de lucro, las relaciones con la industria farmacéutica internacional y la publicación de la mayor parte de sus artículos en revistas editadas en Estados Unidos. Entre las áreas de especialización temática del núcleo se encuentran Farmacología Veterinaria, Toxicología Veterinaria, Fisiología de la Reproducción y Fisiología-Patología del Metabolismo y de la Nutrición; cada una de las cuales tiene su investigador responsable. Sus estrategias de internacionalización se describen y analizan en el próximo apartado.

\footnotetext{
3 De acuerdo con la normativa de la Secretaría de Ciencia, Arte y Tecnología (seCAT), los Núcleos de Actividades Científicas y Tecnológicas son definidos como conjuntos de investigadores y auxiliares dedicados a actividades identificadas por una línea temática, con capacidad para planificar y ejecutar actividades en esa línea, quienes convienen en articular en forma estrecha y perdurable su trabajo, compartiendo espacios físicos, instalaciones, servicios técnicos y administrativos, bajo órganos de gobierno y pautas reglamentarias estipuladas.
} 
Respecto de la metodología de producción de datos, se optó por la técnica de entrevistas semiestructuradas, las cuales fueron realizadas al director del Grupo Veterinarias, que a su vez es responsable del área de Farmacología Veterinaria del Núcleo, a la responsable del área de Toxicología Veterinaria y al responsable del área de Fisiología de la Reproducción. En el caso de Fisiología-Patología del Metabolismo y de la Nutrición, para el cual no fue posible acceder al responsable por encontrarse de licencia, se entrevistó a una investigadora categoría I en el Programa de Incentivos a Docentes-Investigadores de las Universidades Nacionales Argentinas. Es decir, no sólo se consideró la palabra del director del núcleo, sino también la de los distintos responsables de cada una de las secciones que componen al Grupo Veterinarias, esto con el fin de tener una perspectiva más completa acerca del colectivo y sus estrategias de internacionalización. ${ }^{4}$ Cabe indicar que las entrevistas fueron complementadas con documentos institucionales del grupo, como son el Documento Fundacional y las Memorias Académicas. ${ }^{5}$

\section{Las estrategias de internacionalización y los objetivos de la producción de conocimientos en el caso del Grupo Veterinarias}

El grupo de investigación estudiado se propone, por una parte, generar conocimiento científico original con impacto internacional, con el objetivo de tener presencia en la comunidad científica mundial en cada una de las áreas temáticas que componen la estructura científica del núcleo. Por otra parte, procura también producir información técnica de interés regional y na-

4 La selección de los entrevistados se basó en el reconocimiento de las siguientes situaciones: 1) los recursos humanos de los grupos de investigación se hallan organizados en jerarquías y 2) los investigadores más prestigiosos y con mayor experiencia son los que generalmente se dedican a coordinar las políticas de publicación, participación en proyectos de investigación, asistencia a cursos y seminarios, intercambio de investigadores y becarios del grupo (Campanario, 1999), y, en este caso particular, las actividades a desarrollar en el ámbito internacional, contando con una perspectiva histórica y global de la internacionalización del grupo.

5 El Documento Fundacional es un documento entregado por cada NACT reconocido por la SECAT de la Universidad Nacional del Centro de la Provincia de Buenos Aires (UNCPBA) por única vez, en el cual se definen la modalidad de designación de la dirección, las líneas temáticas y las estrategias de desarrollo. Las Memorias Académicas, por su parte, son los informes anuales que tienen que entregar los NACT de la UNCPBA a la SECAT. En ellas se expone la integración actualizada de la planta estable, la formación de recursos humanos, la financiación y desarrollo regular de proyectos de investigación y la producción científica de manera anual. cional con el objetivo de transferirlo rápidamente al medio en el cual se insertan geográficamente.

En principio, vale la pena anotar que la totalidad de los entrevistados destaca la importancia de la formación de los primeros investigadores del grupo en el extranjero, lo cual les permitió consolidar instancias de preparación académica en el ámbito nacional. De este modo, las nuevas generaciones de recursos humanos en formación del Grupo Veterinarias tienen la posibilidad de estudiar su doctorado en la universidad de la cual hacen parte, trasladándose al exterior para instancias de formación posdoctoral. Asimismo, los entrevistados promueven el trabajo con colegas en el extranjero, sobre todo al permitirles el acceso a convocatorias para el desarrollo de proyectos de investigación con contrapartes internacionales, así como al posibilitar sus colaboraciones en revistas editadas en otros países. Además, se hace uso de las nuevas tecnologías de la información y la comunicación para estar en contacto permanente con distintos lugares del mundo y para acceder inmediatamente a nuevos aportes del conocimiento. Por su parte, se considera que el Grupo Veterinarias se esfuerza y llega a posicionarse cercanamente, en lo referido a equipamiento de investigación, a los centros más avanzados, lo cual les permite -al contar con mediciones actualizadas- difundir los resultados de sus investigaciones en el exterior.

Ahora bien, más allá de estas coincidencias, existen desacuerdos al momento de pensar las publicaciones académicas en el exterior y los mecanismos de evaluación de las revistas científicas en relación con los objetivos de producción de conocimientos priorizados por cada uno de los entrevistados. De acuerdo con el director, el Grupo Veterinarias cuenta con una masa crítica de recursos humanos entre los que se encuentran investigadores mayormente dedicados a las actividades de transferencia hacia el medio productivo local y regional e investigadores dedicados al desarrollo de ciencia básica difundida en el ámbito internacional. Particularmente, el director del núcleo y responsable de la especialidad de Farmacología Veterinaria considera que el objetivo central de la producción de conocimientos es la originalidad, característica que se encuentra ligada a la publicación en revistas de corriente principal, lo cual permite la difusión de la ciencia producida por el grupo en la comunidad científica internacional. Por su parte, la entrevistada de la sección de Fisiología-Patología del Metabolismo y de la Nutrición coincide con la perspectiva señalada por el director. 
Según esta concepción, el director del núcleo advierte que las conclusiones del trabajo del área de Farmacología en Medicina veterinaria básica es extrapolado al ámbito de la medicina humana, lo cual les permite participar en proyectos de investigación financiados por instituciones internacionales, como son aquellas dedicadas a la atención de la salud animal y humana de zonas geográficas caracterizadas por la extrema pobreza. En este sentido, se destaca su participación en un proyecto de investigación financiado por una organización escocesa sin fines de lucro para el desarrollo de productos farmacéuticos destinados al control de parásitos problemáticos en África. Igualmente, señala la participación de los integrantes de la rama de Farmacología Veterinaria en un Programa Marco de la Unión Europea en el cual se abordó el control de la Fasciola hepatica, cuya importancia reside en el impacto negativo que este parásito genera en la producción del ganado en los países de la región norte de Europa y de Argentina.

Además, el director del núcleo indica la importancia de sus publicaciones en el exterior como medio de acceso a proyectos conjuntos de investigación con contrapartes extranjeras en tanto las convocatorias evalúan justamente la "calidad" de la investigación a través de indicadores bibliométricos. En este orden de ideas, se observa la puesta en marcha de un "círculo virtuoso" por el cual el prestigio de base obtenido por el reconocimiento de la producción científica local en el marco de las revistas de corriente principal, funciona como elemento legitimador de los investigadores del núcleo para la participación en proyectos conjuntos con actores de otros países. La investigadora del área de Fisiología-Patología del Metabolismo y de la Nutrición entrevistada destaca la importancia del contacto con laboratorios en el extranjero como medio para acceder a mediciones no disponibles en el contexto nacional, pero necesarias para acceder a publicaciones en revistas del mainstream científico.

Por otro lado, la responsable de la especialidad de Toxicología Veterinaria considera que el objetivo principal de su actividad de investigación es la transferencia de conocimientos al medio productivo y social, en pos de la resolución de problemáticas surgidas del contexto en el que se inserta el grupo. De esta manera, si bien resalta la "calidad" de los papers publicados en las revistas de corriente principal, relativiza el concepto de "impacto" asociado a los indicadores bibliométricos.
De acuerdo con la entrevistada, estos indicadores pueden asegurar la calidad lógica y metodológica de los artículos académicos; sin embargo, no pueden evaluar la relevancia de los aportes para la resolución de problemas productivos y sociales, lo cual implicaría la utilización de indicadores diferentes de evaluación, así como también una diversificación en la conformación de los comités evaluadores.

Coincidentemente, el responsable de la sección de Fisiología de la Reproducción manifiesta que las publicaciones en el extranjero son consideradas productos secundarios desprendidos del objetivo central del área, a saber, dar respuestas a las problemáticas reproductivas del medio. Para ejemplificar esta concepción de la rama de Reproducción respecto de la internacionalización de la publicación científica, el entrevistado indica el caso de las investigaciones referidas a la reproducción del Myocastor coypus, animal más comúnmente conocido como nutria. Así, el responsable de esta especialidad señala que el estudio de dicha temática surgió en respuesta a la demanda de los productores locales abocados a la cría de nutrias. Paralelamente, las publicaciones surgidas de los estudios sobre la reproducción del animal fueron ampliamente aceptadas en el extranjero por la ausencia de aportes al respecto en la comunidad científica internacional. Sin embargo, una vez que la demanda de los productores locales cambió de rumbo, el Grupo Veterinarias abandonó los estudios sobre el Myocastor coypus, más allá de que estos constituyeran una "mina de oro" en lo que a internacionalización de la publicación se refiere.

Por otra parte, el responsable de Fisiología de la Reproducción resalta que las publicaciones en revistas internacionales, si bien permiten difundir la producción del grupo en la comunidad científica, difícilmente les permiten llegar a los colegas veterinarios del ámbito privado, destinatarios de los esfuerzos de investigación en el área. De acuerdo con esta percepción, si bien cuenta con publicaciones en el extranjero, indexadas en el Institute for Scientific Information (ISI), la producción científica más fuerte de la sección se encuentra en el ámbito nacional. De esta manera, se busca que el colega veterinario inmerso en la actividad privada tenga acceso a información actualizada, validada y probada sobre metodologías tendientes a la eficiencia reproductiva, desarrolladas por el núcleo y difundidas en circuitos nacionales de publicación. 
Asimismo, cabe señalar que el responsable de la rama de Fisiología de la Reproducción se muestra crítico ante los conceptos y tecnologías que se comunican en las reuniones internacionales a las que asiste o que se adquieren en las estadías de perfeccionamiento realizadas en el exterior, ya que estos pueden no ser adecuados para la solución de problemáticas reproductivas y productivas del contexto regional y nacional en el que se halla inserto el grupo. De acuerdo con el entrevistado, el principal objetivo de la especialidad de Reproducción es responder a las demandas del colega veterinario, con lo cual la "importación" de conceptos generados en otros contextos requiere una "vigilancia" respecto de su aplicabilidad en el medio local.

Las diferentes entrevistas realizadas dan cuenta de la tensión resaltada por Kreimer (2006) entre la preocupación por reforzar los vínculos con el uso social del conocimiento en un contexto local y la vocación de legitimación de los conocimientos en la comunidad internacional de pares de un campo determinado, la cual, en el caso del Grupo Veterinarias, se intenta salvar mayormente a partir de distintas estrategias de articulación entre los objetivos de la producción de conocimiento en relación con la orientación de la investigación descrita en el apartado siguiente. Dichas estrategias son:

- La división del trabajo dentro del núcleo, por la cual algunos investigadores desarrollan mayormente tareas de difusión en el ámbito internacional, y otros, tareas de transferencia de conocimientos al medio productivo.

- La división de tareas entre investigadores formados y en formación, por la cual los investigadores de mayor trayectoria atienden a las demandas de las empresas, mientras que los becarios estudian los aspectos fundamentales de las temáticas planteadas desde el sector privado, las cuales se insertan con mayor facilidad en el ámbito internacional.

- El aprovechamiento de proyectos aplicados para la aproximación a temas ligados con preocupaciones teóricas generales, las cuales resultan importantes para explicar los problemas del medio y para la comunidad de pares, aunque no imprescindibles desde el punto de vista de los productores, que buscan soluciones puntuales y concretas.

- La complementación entre los servicios prestados al sector privado y el desarrollo de teorías basadas en casos que puedan ser difundidas en el ámbito internacional.
Estas estrategias se relacionan, igualmente, con las exigencias institucionales que los actores entrevistados consideran que existen sobre su actividad de investigación, lo cual se analiza en el siguiente apartado.

\section{Las estrategias de internacionalización y la orientación de la investigación en el caso del grupo Veterinarias}

En el ámbito científico-tecnológico público-estatal, el Grupo Veterinarias posee una heterogénea afiliación institucional, distinguiéndose tres ámbitos de evaluación: a) los organismos estatales que cuentan con becas de formación y carrera científica - como el Consejo Nacional de Investigaciones Científicas y Técnicas (CONICET) y la Comisión de Investigaciones Científicas de la Provincia de Buenos Aires (CICPBA) - y con subsidios para la investigación y desarrollo $(\mathrm{I}+\mathrm{D})-$ como la Agencia Nacional de Promoción Científica y Tecnológica (ANPCYT)—; b) las políticas propias que implementa la universidad y que no están asociadas a programas u organismos externos; y c) el Programa de Incentivos a los Docentes-Investigadores de Universidades Nacionales del Ministerio de Educación de la Nación. Cada uno de estos organismos evalúa la actividad investigativa del núcleo. Si bien existen normativas y discursos oficiales acerca de los objetivos y pautas de evaluación primordiales en cada caso, este trabajo indaga la manera particular en que el grupo considera que los criterios de evaluación influyen en la internacionalización del núcleo, así como explora las estrategias que desarrollan en consonancia con estos.

Desde su experiencia como evaluador e integrante de la carrera de investigador del CONICET, el director del Grupo Veterinarias afirma que los criterios de evaluación del Consejo priorizan la cantidad de publicaciones científicas realizadas por sus investigadores, así como también las citas recibidas por parte de los colegas. En el mismo sentido, la responsable del área de Toxicología Veterinaria advierte que las evaluaciones de CONICET ponderan fundamentalmente los artículos científicos publicados en el ámbito internacional, lo cual guía su comportamiento respecto de la publicación científica. Más precisamente, la entrevistada considera que si bien es posible identificar políticas institucionales formales, es en las comisiones evaluadoras el espacio en que concretamente se lleva a cabo la evaluación; allí se sigue prestando atención a la cantidad de papers indexados y el factor de impacto de estos. 
El director del Grupo Veterinarias asegura que el trabajo del núcleo se halla relacionado fuertemente con CONICET, ${ }^{6}$ aunque también se ha contado con el apoyo de la Сісрва. ${ }^{7} \mathrm{Al}$ respecto de los criterios de selección de proyectos para su financiamiento por parte de esta última entidad, el responsable de Fisiología de la Reproducción indica la existencia de subsidios específicos destinados a promover la resolución de problemáticas de la provincia a través de la producción de conocimiento. Por otra parte, el investigador expresa su acuerdo con este tipo de subsidios que orientan la producción de conocimiento hacia la resolución de problemáticas del medio productivo, a la vez que informa la poca frecuencia con que se presentan estas iniciativas. De acuerdo con las palabras del investigador, la sección en la cual desarrolla su trabajo dentro del Grupo Veterinarias presenta las características necesarias para obtener este tipo de subsidios dirigidos a la atención de las demandas productivas. Por otra parte, el director del núcleo manifiesta que la СІСРвA ha ido perdiendo, a lo largo de la trayectoria del Grupo Veterinarias, el protagonismo que supo tener en los orígenes de este, siendo CONICET y la Agencia Nacional de Promoción Científica y Tecnológica las principales fuentes de financiamiento actual del grupo. ${ }^{8}$

Al respecto, la responsable de Toxicología Veterinaria señala que tanto la creación de la Agencia como su integración al Ministerio de Ciencia, Tecnología e Innovación Productiva, creado en el 2007, han representado un cambio en la orientación de la investigación en Argentina, al darle mayor peso al financiamiento de la investigación tecnológica, alentar la presentación de iniciativas conjuntas entre empresas y grupos de investigación, y priorizar problemáticas regionales. Sin embargo, advierte una vez más que si bien existen di-

\footnotetext{
6 El Consejo Nacional de Investigaciones Científicas y Técnicas data desde 1958 y es un organismo dependiente del Ministerio de Ciencia, Tecnología e Innovación Productiva, que ejecuta, fomenta y financia la investigación científica y tecnológica y las actividades de apoyo que apunten al avance científico y tecnológico en el país. Para ello, cuenta con institutos de investigación propios, con un sistema de becas y subsidios a proyectos, así como también con una carrera de investigador.

La Comisión de Investigaciones Científicas de la Provincia de Buenos Aires fue creada en 1968 y se propone fomentar y financiar las actividades de ciencia y tecnología desarrolladas en territorio de la provincia de Buenos Aires.

8 La Agencia Nacional de Promoción Científica y Tecnológica es un organismo nacional creado en 1996 y dependiente, desde su fundación en 2007, del Ministerio de Ciencia, Tecnología e Innovación Productiva de Argentina; está dedicado a promover el financiamiento de proyectos de investigación y de innovación tecnológica tendientes a mejorar las condiciones sociales, económicas y culturales en el país.
}

ferentes políticas de acuerdo al organismo de Ciencia y Tecnología de que se trate, en última instancia la evaluación se define a partir del criterio subjetivo de quienes conforman los comités de pares. Además, reconoce la dificultad que tiene el sistema científico-tecnológico argentino para evaluar el "impacto" de la producción de conocimiento en otros términos que no sea a través del "factor de impacto" de las revistas científicas.

Por otro lado, el responsable de Reproducción asevera que, en términos generales, la evaluación de los investigadores dentro del sistema académico tiene en cuenta las publicaciones en revistas de corriente principal, con lo cual dicho tipo de publicación se vuelve una exigencia central para ellos, más allá de los objetivos del área respecto a la atención de las demandas de los productores agropecuarios del ámbito regional y nacional. En este marco, el responsable de la especialidad de Reproducción afirma que la priorización de las publicaciones indexadas en el IsI y el Sistema Regional de Información en Línea para Revistas Científicas de América Latina, el Caribe, España y Portugal (LATINDEX) se extiende cada vez más a diferentes instituciones, entre las que se destaca la Secretaría de Ciencia, Arte y Tecnología de la UnCPBA. De este modo, en la Universidad Nacional del Centro se ve una tendencia a brindar mayor importancia a la publicación de la producción realizada por los docentes-investigadores que trabajan en ella en revistas indexadas según la evaluación de la calidad científica.

Ahora bien, el responsable de la rama de Reproducción, quien se desempeña como evaluador de la SECAT, relativiza la orientación de la ciencia y la tecnología producida en la universidad únicamente hacia las publicaciones en revistas indexadas del ámbito internacional. El entrevistado indica que, desde su rol de evaluador, intenta valorar todas las actividades llevadas a cabo por los grupos de investigación de la institución de acuerdo con los objetivos que cada uno de ellos se propone. Así mismo, destaca que varios colegas evaluadores poseen la misma visión sobre la valoración de las actividades de ciencia y tecnología, con lo cual las perspectivas individuales, mantenidas a lo largo de los años, se van transformando en "verdaderas políticas institucionales", según palabras del entrevistado. De esta manera, surge nuevamente la distinción entre la política institucional y los criterios de evaluación aplicados por las comisiones de pares evaluadores, esta vez para relativizar el peso de las publicaciones indexadas en las evaluaciones llevadas a cabo en el marco de la SECAT. 
Respecto del Programa de Incentivos a Docentes-Investigadores de las Universidades Nacionales, el responsable de Reproducción considera que evalúa la producción científica, la formación de recursos humanos y la extensión. En la misma línea, afirma que los resultados de las evaluaciones del núcleo en el Programa de Incentivos han sido positivos, ya que "el grupo hace todo", es decir, responden a los distintos requisitos planteados por las evaluaciones científico-tecnológicas. Por su parte, la investigadora del área de Fisiología y Patología del Metabolismo y de la Nutrición advierte que la publicación en revistas extranjeras se convierte en una exigencia para mantenerse en el Programa de Incentivos.

Respecto al financiamiento, el Grupo Veterinarias cuenta con fondos provenientes de rutinas de recursos y dispositivos de recursos construidos por el grupo de investigación. En el caso de las rutinas de recursos, el colectivo recibe subsidios anuales otorgados por la propia universidad, los cuales se calculan con base en los recursos externos obtenidos por el grupo, provenientes del ámbito nacional e internacional, público y privado. Por su parte, las agencias que financian la mayor parte de los salarios y proyectos de investigación del grupo son el CONICET, la CICPBA y la ANPCYT. Además, los entrevistados reconocen el aumento del gasto público en ciencia y tecnología y la diversificación de las fuentes de financiamiento en el ámbito público-estatal producido en Argentina en los últimos años.

Ahora bien, de acuerdo con los testimonios relevados, el Grupo Veterinarias requiere de la complementación de estas rutinas de recursos con ciertos dispositivos de recursos generados por el grupo tanto en el ámbito nacional como internacional, en el público como en el privado. Esto se vincula con la necesidad de adquirir recursos extra que permitan costear el equipamiento, el service de los equipos, los repuestos, los accesorios, el material de consumo, los solventes, los gases, los animales para experimentación y el alimento, cuestiones centrales para el trabajo de investigación del núcleo.

De esta manera, se tiene en cuenta el desarrollo de estrategias de complementación entre los recursos nacionales y extranjeros, reconociendo el aumento y diversificación de las fuentes nacionales de financiamiento en los últimos años así como también la importancia de los pagos en moneda extranjera obtenidos en el ámbito internacional. En tal sentido, las estrategias desarrolladas consisten en la postulación a con- vocatorias de proyectos conjuntos de investigación y la prestación de servicios a empresas farmacéuticas internacionales.

\section{Reflexiones finales sobre las relaciones entre objetivos de investigación, orientación de la investigación y las estrategias de internacionalización científica}

El presente artículo se propuso contribuir al análisis de la internacionalización científica de los grupos de investigación, haciendo especial hincapié en las estrategias de internacionalización y entendiendo que estas influyen en la producción de conocimiento y en los procesos de interpretación y delineamiento de las políticas públicas en ciencia y tecnología. Una de las dimensiones analizadas se refiere las estrategias de internacionalización desarrolladas en relación con los objetivos que tiene la producción de conocimientos para los integrantes de los grupos de investigación. Otra de las dimensiones estudiadas se refiere a las estrategias de internacionalización desarrolladas en relación con los procesos de evaluación y financiamiento de la ciencia y la tecnología llevados adelante por los grupos de investigación.

En términos generales, el grupo de investigación seleccionado para el estudio entiende que la producción de conocimientos tiene que estar orientada tanto al ámbito internacional como local. Sin embargo, los actores entrevistados se identifican más fuertemente con un ámbito que con otro, dando un sentido diferencial al proceso de internacionalización y a las estrategias desarrolladas al respecto. Así, mientras algunos tienen como meta el desarrollo de actividades en el contexto internacional que permitan visibilizar su producción de conocimientos y sus distintas capacidades como grupo de investigación, otros consideran la internacionalización como un subproducto de sus tareas orientadas a la resolución de problemáticas productivas locales. En su conjunto y dentro de las áreas, el Grupo Veterinarias desarrolla estrategias de articulación entre los distintos objetivos de la producción de conocimiento y las exigencias institucionales de evaluación.

Por su parte, son las instituciones de ciencia y tecnología que enmarcan el trabajo del grupo las que orientan la actividad de investigación y exigen, fundamentalmente, la internacionalización de la producción científica, lo cual permite explicar igualmente las estra- 
tegias de articulación puestas en marcha por el Grupo Veterinarias. En cuanto a las fuentes de financiamiento para la investigación llevada adelante por el grupo, se destacan las estrategias de complementación, divididas entre las rutinas de recursos del ámbito nacional y los dispositivos generados en el ámbito internacional a través de la postulación a proyectos conjuntos de investigación y la prestación de servicios.

A su vez, si bien todos reconocen que las evaluaciones científicas y la necesidad de obtener recursos adicionales influyen en la decisión del grupo de internacionalizar su producción de conocimiento y su búsqueda de fuentes de financiamiento, las distintas visiones sobre los objetivos de la producción de conocimiento $-\mathrm{y}$ sus estrategias- influyen en los espacios de evaluación protagonizados por los actores entrevistados. En dichas instancias, cada uno, en tanto que evaluador, pone en juego sus visiones sobre la internacionalización, contribuyendo a la orientación de la investigación, a la vez que se es influido por esta en la tarea cotidiana de producción de conocimientos. De esta manera, es necesario tener en cuenta el papel de los investigadores en las instancias de delineamiento e interpretación de las políticas en ciencia y tecnología.

Cabe señalar que los actores tienden a valorar positivamente lo que "saben hacer mejor", poniendo un realce mayor en la realización de las actividades que se valoran positivamente, sin desatender el "deber" internacionalizarse, supuesto en el sistema nacional de evaluación de las actividades científicas y tecnológicas y en los mecanismos de financiamiento del grupo. Asimismo, es en la brecha que existe entre las políticas explícitas institucionales y las políticas llevadas efectivamente adelante por los pares evaluadores, donde se pone en juego lo que se sabe hacer mejor y, por lo tanto, lo que se valora positivamente en la actividad científica. En este punto, salta a la luz el juego de mantenimiento de prestigio por parte de los investigadores a partir de la definición de la actividad científica y del papel de la internacionalización en esta.

Se considera fundamental, como líneas futuras de investigación, atender a los procesos que explican la conformación de diferentes estrategias de internacionalización, analizando, por ejemplo, el papel de las disciplinas y las distintas trayectorias de los investigadores. Se entiende que la prosecución de trabajos de corte empírico puede revelar dimensiones importantes que escapan al marco de análisis propuesto. Además, se entiende que la apertura del análisis de las estrategias de los grupos de investigación hacia otras dimensiones de la producción de conocimiento más amplias que, aunque atravesadas por la internacionalización (como, por ejemplo, la estipulación de las agendas de investigación, la generación de relaciones de recursos o la difusión de los conocimientos producidos), puede contribuir a complejizar el análisis sobre la investigación y su relación con las políticas públicas en ciencia y tecnología.

\section{Referencias}

Albornoz, M. (2009). Desarrollo y políticas públicas en ciencia y tecnología en América Latina. RIPS. Revista de Investigaciones Políticas y Sociológicas, 8(1). 65-75.

Bianco, M. y Sutz, J. (2005). Las formas colectivas de la investigación universitaria. Revista Iberoamericana de Ciencia, Tecnología y Sociedad- CTS, 2(6), 25-44.

Bourdieu, P. (1987). De la regla a las estrategias. Cosas dichas (pp. 67-83). Buenos Aires: Gedisa.

Campanario, J. M. (1999). La ciencia que no enseñamos. Enseñanza de las Ciencias, 17(3), 397-410.

Dagnino, R. (2007). ¿Cómo participa la comunidad de investigación en la política de C\&T y en la Educación Superior? Educación Superior y Sociedad, 12(1), 1-41.

De Filippo, D., Morillo, F. y Fernández, M. T. (2008). Indicadores de colaboración científica del csic con Latinoamérica en bases de datos internacionales. Revista Española de Documentación Científica, 31(1), 66-84.

Gaillard, J. F. (1994). North-South Research Partnership: Is collaboration possible between Unequal Partners. Knowledge, Technology \& Policy, 7(2), 31-63.

Hubert, M. y Spivak, A. (2009). Integrarse en redes de cooperación en nanociencias y nanotecnologías: el rol de los dispositivos instrumentales. Redes, 15(29), 69-91.

Kreimer, P. (2000). ¿Una modernidad periférica? La investigación científica entre el universalismo y el contexto. En D. Obregón (Ed.), Culturas científicas y saberes locales (pp. 163-196). Bogotá: Universidad Nacional de Colombia.

Kreimer, P. (2006). ¿Dependientes o integrados? La ciencia latinoamericana y la división internacional del trabajo. Nómadas, 24, 199-212.

Kreimer, P. y Ugartemendía, V. (2007). Ciencia en la universidad: dimensiones locales e internacionales. Atos de Pesquisa em Educação, 2(3), 461-485.

Kreimer, P. y Levin, L. (2011). Mapping trends and patterns in S\&T Cooperation between the European union and Latin American countries based on FP6 and FP7 projects. En J. Gaillard y R. Arvanitis (Eds.), Mapping and understanding Science and technology collaboration between Europe and Latin America. Bolivia: L'Institut de Recherche pour le Développement. 
Licha, I. (1996). La globalización de la investigación académica en América Latina. En M. Albornoz, P. Kreimer y E. Glavich (Eds.), Ciencia y sociedad en América Latina. Buenos Aires: Universidad Nacional de Quilmes.

Luchilo, L. y Guber, R. (2007). La infraestructura para la investigación universitaria en Argentina. Educación Superior y Sociedad, 12(1), 114-131.

Plaza, L. M. y Bordons, M. (2006). Proyección internacional de la ciencia española. Anuario del Instituto Cervantes, 547-567.

Red Iberoamericana de Indicadores de Ciencia y Tecnología (RICYT). (2007). Manual de Indicadores de Internacionalización de la Ciencia y de la Tecnología. Manual de Santiago. Recuperado de: http://www.oei.es/salactsi/ manual_santiago.pdf

Sebastián, J. (2003). Estrategias de cooperación universitaria para la formación de investigadores en Iberoamérica. Madrid: Organización de Estados Iberoamericanos para la Educación, la Ciencia y la Cultura (OEI).

Vaccarezza, L. (2000). Las estrategias de desempeño de la profesión académica. Ciencia periférica y sustentabilidad del rol de investigador universitario. Redes, 7(15), 15-43.
Vaccarezza, L. S. y Zabala, J. P. (2002). La construcción de la utilidad social de la ciencia: Investigadores en biotecnología frente al mercado. Buenos Aires: Universidad Nacional de Quilmes.

Vessuri, H. (1991). Universalismo y nacionalismo en la ciencia moderna. Una aproximación desde el caso venezolano. Quipu, 8(2), 255-271.

Vessuri, H. (1998). La movilidad científica desde la perspectiva de América Latina. En J. B. Meyer y J. Charum (Eds.), El nuevo nomadismo científico: la perspectiva latinoamericana (pp. 99-113). Colombia: Escuela Superior de Investigación Pública (ESIP).

Vessuri, H. (2009). Cambios Recientes en la internacionalización de las ciencias sociales: la sociedad de redes impacta América Latina. En S. Didou y E. Gérard (Eds.), Fuga de cerebros, movilidad académica y redes científicas: perspectiva latinoamericana (pp. 189-203). México: Iesalc - Cinvestav - IRD.

Wilkis, A. (2004). Apuntes sobre la noción de estrategia en Pierre Bourdieu. Revista Argentina de Sociología, 3, 118130. 